\title{
On the Rotation of a Vector Field in a Four-Dimensional Space
}

\author{
Leonardo Simal Moreira \\ UniFOA —Centro Universitário de Volta Redonda, Volta Redonda, Brazil \\ Email: simal.leonardo@terra.com.br, leonardo.moreira@foa.org.br
}

Received November 5, 2013; revised December 5, 2013; accepted December 12, 2013

Copyright (C) 2014 Leonardo Simal Moreira. This is an open access article distributed under the Creative Commons Attribution License, which permits unrestricted use, distribution, and reproduction in any medium, provided the original work is properly cited. In accordance of the Creative Commons Attribution License all Copyrights (C) 2014 are reserved for SCIRP and the owner of the intellectual property Leonardo Simal Moreira. All Copyright (C) 2014 are guarded by law and by SCIRP as a guardian.

\section{ABSTRACT}

Recently I published a paper in the journal ALAMT (Advances in Linear Algebra \& Matrix Theory) and explored the possibility of obtaining products of vectors in dimensions higher than three [1]. In continuation to this work, it is proposed to develop, through dimensional analogy, a vector field with notation and properties analogous to the curl, in this case applied to the space $I R^{4}$. One can see how the similarities are obvious in relation to the algebraic properties and the geometric structures, if the rotations are compared in spaces of three and four dimensions.

\section{KEYWORDS}

Products of Vectors; Dimensional Analogy; Vector Fields; Curl; Rotations; Curl by Analogy

\section{Introduction}

[1] has defined a similar operation to the cross product, to a space of dimension $n$. In particular, for a 4-dimensional Cartesian space with Euclidean norm, we have the following results:

Given three linearly independent vectors, $\boldsymbol{F}_{1}, \boldsymbol{F}_{2}$ and $\boldsymbol{F}_{3}$, such that $\boldsymbol{F}_{i}=m_{i} \hat{e}_{1}+n_{i} \hat{e}_{2}+p_{i} \hat{e}_{3}+q_{i} \hat{e}_{4}$, then:

$$
\left[\boldsymbol{F}_{1} \boldsymbol{F}_{2} \boldsymbol{F}_{3}\right]=\left|\begin{array}{cccc}
\hat{e}_{1} & \hat{e}_{2} & \hat{e}_{3} & \hat{e}_{4} \\
m_{1} & n_{1} & p_{1} & q_{1} \\
m_{2} & n_{2} & p_{2} & q_{2} \\
m_{3} & n_{3} & p_{3} & q_{3}
\end{array}\right|,
$$

being

$$
\left[\boldsymbol{F}_{1} \boldsymbol{F}_{2} \boldsymbol{F}_{3}\right] \cdot \boldsymbol{F}_{i}=0 \quad(i=1,2,3) .
$$

The relationship between the norms of the vectors $\boldsymbol{F}_{i}$ is given by:

$$
\left|\left[\boldsymbol{F}_{1} \boldsymbol{F}_{2} \boldsymbol{F}_{3}\right]\right|=\left|\boldsymbol{F}_{1}\right|\left|\boldsymbol{F}_{2}\right|\left|\boldsymbol{F}_{3}\right| k,
$$

where

$$
k=\left|\begin{array}{ccc}
1 & \cos \alpha_{12} & \cos \alpha_{13} \\
\cos \alpha_{21} & 1 & \cos \alpha_{23} \\
\cos \alpha_{31} & \cos \alpha_{32} & 1
\end{array}\right|^{\frac{1}{2}} .
$$

In (4), $\alpha_{i j}(i, j=1,2,3)$ represents the angles between two vectors that generate the product $\left[\boldsymbol{F}_{1} \boldsymbol{F}_{2} \boldsymbol{F}_{3}\right]$, with 
conditions established in [1].

\section{Basic Properties of Vector Operators in Four Dimensions}

Be summarized important properties about vector operators, here extended to four dimensions, with the objective of situate the reader on what is intended to present in Section 3. In these results, it is considered that $u_{i}=u_{i}\left(x_{1}, x_{2}, x_{3}, x_{4}\right)$ and $\boldsymbol{F}_{i}=\boldsymbol{F}_{i}\left(x_{1}, x_{2}, x_{3}, x_{4}\right), i=1, \cdots, n$, respectively represent scalar fields and vector fields on $I R^{4}$, with defined partial derivatives.

\subsection{The Del Operator in Four Dimensions}

$$
\nabla=\hat{e}_{1} \frac{\partial}{\partial x_{1}}+\hat{e}_{2} \frac{\partial}{\partial x_{2}}+\hat{e}_{3} \frac{\partial}{\partial x_{3}}+\hat{e}_{4} \frac{\partial}{\partial x_{4}},
$$

being $\hat{e}_{1}=(1,0,0,0), \hat{e}_{2}=(0,1,0,0), \hat{e}_{3}=(0,0,1,0)$ and $\hat{e}_{4}=(0,0,0,1)$.

\subsection{The Gradient in Four Dimensions}

$$
\operatorname{grad} u_{i}=\nabla u_{i}=\frac{\partial u_{i}}{\partial x_{1}} \hat{e}_{1}+\frac{\partial u_{i}}{\partial x_{2}} \hat{e}_{2}+\frac{\partial u_{i}}{\partial x_{3}} \hat{e}_{3}+\frac{\partial u_{i}}{\partial x_{4}} \hat{e}_{4}
$$

It is valid the following elementary property:

$$
\nabla\left(\sum_{i=1}^{n} u_{i}\right)=\sum_{i=1}^{n}\left(\nabla u_{i}\right)
$$

\subsection{The Divergence in Four Dimensions}

$$
\operatorname{div} \boldsymbol{F}_{i}=\nabla \cdot \boldsymbol{F}_{i}=\frac{\partial m_{i}}{\partial x_{1}}+\frac{\partial n_{i}}{\partial x_{2}}+\frac{\partial p_{i}}{\partial x_{3}}+\frac{\partial q_{i}}{\partial x_{4}} ;(i=1, \cdots, n),
$$

considering that $\boldsymbol{F}_{i}=m_{i} \hat{e}_{1}+n_{i} \hat{e}_{2}+p_{i} \hat{e}_{3}+q_{i} \hat{e}_{4}$, and further $m_{i}=m_{i}\left(x_{1}, x_{2}, x_{3}, x_{4}\right), \quad n_{i}=n_{i}\left(x_{1}, x_{2}, x_{3}, x_{4}\right)$, $p_{i}=p_{i}\left(x_{1}, x_{2}, x_{3}, x_{4}\right)$ and $q_{i}=q_{i}\left(x_{1}, x_{2}, x_{3}, x_{4}\right)$.

It is valid the following elementary property:

$$
\nabla \cdot\left(\sum_{i=1}^{n} \boldsymbol{F}_{i}\right)=\sum_{i=1}^{n}\left(\nabla \cdot \boldsymbol{F}_{i}\right)
$$

\subsection{Relationship between the Gradient and the Divergence}

$$
\nabla \cdot\left(u_{i} \boldsymbol{F}_{j}\right)=\left(\nabla u_{i}\right) \cdot \boldsymbol{F}_{j}+u_{i}\left(\nabla \cdot \boldsymbol{F}_{j}\right), \quad\left\{\begin{array}{l}
i=1, \cdots, m \\
j=1, \cdots, n
\end{array}\right.
$$

\subsection{The Laplacian in Four Dimensions}

\subsubsection{The Laplacian of $u_{i}$}

$$
\nabla^{2} u_{i}=\frac{\partial^{2} u_{i}}{\partial x_{1}^{2}}+\frac{\partial^{2} u_{i}}{\partial x_{2}^{2}}+\frac{\partial^{2} u_{i}}{\partial x_{3}^{2}}+\frac{\partial^{2} u_{i}}{\partial x_{4}^{2}} ;(i=1, \cdots, n)
$$

2.5.2. The Laplacian of $F_{i}$

$$
\nabla^{2} \boldsymbol{F}_{i}=\frac{\partial^{2} \boldsymbol{F}_{i}}{\partial x_{1}^{2}}+\frac{\partial^{2} \boldsymbol{F}_{i}}{\partial x_{2}^{2}}+\frac{\partial^{2} \boldsymbol{F}_{i}}{\partial x_{3}^{2}}+\frac{\partial^{2} \boldsymbol{F}_{i}}{\partial x_{4}^{2}} ;(i=1, \cdots, n),
$$




\section{Curl by Analogy in IR}

It is proposed to introduce an analog curl in $I R^{4}$. Given two vector fields in $I R^{4}$, represented by $\boldsymbol{F}_{1}=m_{1} \hat{e}_{1}+n_{1} \hat{e}_{2}+p_{1} \hat{e}_{3}+q_{1} \hat{e}_{4}$ and $\boldsymbol{F}_{2}=m_{2} \hat{e}_{1}+n_{2} \hat{e}_{2}+p_{2} \hat{e}_{3}+q_{2} \hat{e}_{4}$, consider the vector product

$$
\begin{aligned}
\Psi & =\left[\nabla \boldsymbol{F}_{1} \boldsymbol{F}_{2}\right]=\left[\frac{\partial}{\partial x_{2}}\left(p_{1} q_{2}-q_{1} p_{2}\right)+\frac{\partial}{\partial x_{3}}\left(q_{1} n_{2}-n_{1} q_{2}\right)+\frac{\partial}{\partial x_{4}}\left(n_{1} p_{2}-p_{1} n_{2}\right)\right] \hat{e}_{1} \\
& -\left[\frac{\partial}{\partial x_{1}}\left(p_{1} q_{2}-q_{1} p_{2}\right)+\frac{\partial}{\partial x_{3}}\left(q_{1} m_{2}-m_{1} q_{2}\right)+\frac{\partial}{\partial x_{4}}\left(m_{1} p_{2}-p_{1} m_{2}\right)\right] \hat{e}_{2} \\
& +\left[\frac{\partial}{\partial x_{1}}\left(n_{1} q_{2}-q_{1} n_{2}\right)+\frac{\partial}{\partial x_{2}}\left(q_{1} m_{2}-m_{1} q_{2}\right)+\frac{\partial}{\partial x_{4}}\left(m_{1} n_{2}-n_{1} m_{2}\right)\right] \hat{e}_{3} \\
& -\left[\frac{\partial}{\partial x_{1}}\left(n_{1} p_{2}-p_{1} n_{2}\right)+\frac{\partial}{\partial x_{2}}\left(p_{1} m_{2}-m_{1} p_{2}\right)+\frac{\partial}{\partial x_{3}}\left(m_{1} n_{2}-n_{1} m_{2}\right)\right] \hat{e}_{4}
\end{aligned}
$$

It is important to note that this definition provides for the inclusion of two vector fields simultaneously in the calculations.

In the remainder of this paper, the vector field $\Psi=\left[\nabla \boldsymbol{F}_{1} \boldsymbol{F}_{2}\right]$ is identified by the symbol $\nabla \times\left(\boldsymbol{F}_{1}, \boldsymbol{F}_{2}\right)$, where $\left(\boldsymbol{F}_{1}, \boldsymbol{F}_{2}\right)$ is related to the ordered nature of the product of vector, and $\times$ characterizes its vectorial aspect (however, it should be noted that there is no relation with the usual cross product, defined in $I R^{3}$ ).

In compact notation, $\nabla \times\left(\boldsymbol{F}_{1}, \boldsymbol{F}_{2}\right)$ may be represented by the symbolic determinant

$$
\nabla \times\left(\boldsymbol{F}_{1}, \boldsymbol{F}_{2}\right)=\left|\begin{array}{cccc}
\hat{e}_{1} & \hat{e}_{2} & \hat{e}_{3} & \hat{e}_{4} \\
\frac{\partial}{\partial x_{1}} & \frac{\partial}{\partial x_{2}} & \frac{\partial}{\partial x_{3}} & \frac{\partial}{\partial x_{4}} \\
m_{1} & n_{1} & p_{1} & q_{1} \\
m_{2} & n_{2} & p_{2} & q_{2}
\end{array}\right| .
$$

The analogy with the curl is based on a symbolic notation, obtained based on structure of determinants and their relationship with the vector "Del”. In subsequent section will be shown how this vector thus defined is associated to the rotational motion in four-dimensional space.

Rotational motion, here and in the remainder of this paper, should not be interpreted as a movement with real physical meaning, but only in the context of geometric relations between vectors.

\section{Properties of the Vector $\nabla \times\left(F_{1}, F_{2}\right)$}

Adding to the basic properties (P1)-(P3) involving $\nabla$, which are valid in any dimension, presents the following properties associated with the vector $\nabla \times\left(\boldsymbol{F}_{1}, \boldsymbol{F}_{2}\right)$ and valid in space $I R^{4}$ :

$$
\nabla \times\left(\boldsymbol{F}_{1}+\boldsymbol{F}_{2}, \boldsymbol{F}_{3}\right)=\nabla \times\left(\boldsymbol{F}_{1}, \boldsymbol{F}_{3}\right)+\nabla \times\left(\boldsymbol{F}_{2}, \boldsymbol{F}_{3}\right)
$$

Corollary:

$$
\begin{aligned}
& \nabla \times\left(\boldsymbol{F}_{1}, \boldsymbol{F}_{2}+\boldsymbol{F}_{3}\right)=\nabla \times\left(\boldsymbol{F}_{1}, \boldsymbol{F}_{2}\right)+\nabla \times\left(\boldsymbol{F}_{1}, \boldsymbol{F}_{3}\right) \\
& \nabla \times\left(\boldsymbol{F}_{1}+\boldsymbol{F}_{2}, \boldsymbol{F}_{3}+\boldsymbol{F}_{4}\right)=\nabla \times\left(\boldsymbol{F}_{1}, \boldsymbol{F}_{3}\right)+\nabla \times\left(\boldsymbol{F}_{2}, \boldsymbol{F}_{3}\right)+\nabla \times\left(\boldsymbol{F}_{1}, \boldsymbol{F}_{4}\right)+\nabla \times\left(\boldsymbol{F}_{2}, \boldsymbol{F}_{4}\right) \\
& \nabla \times\left(u_{i} \boldsymbol{F}_{1}, \boldsymbol{F}_{2}\right)=\left[\left(\nabla u_{i}\right) \boldsymbol{F}_{1} \boldsymbol{F}_{2}\right]+u_{i}\left(\nabla \times\left(\boldsymbol{F}_{1}, \boldsymbol{F}_{2}\right)\right) \\
& \nabla \cdot\left[\boldsymbol{F}_{1} \boldsymbol{F}_{2} \boldsymbol{F}_{3}\right]=-\boldsymbol{F}_{1} \cdot \nabla \times\left(\boldsymbol{F}_{2}, \boldsymbol{F}_{3}\right)+\boldsymbol{F}_{2} \cdot \nabla \times\left(\boldsymbol{F}_{1}, \boldsymbol{F}_{3}\right)-\boldsymbol{F}_{3} \cdot \nabla \times\left(\boldsymbol{F}_{1}, \boldsymbol{F}_{2}\right) \\
& \nabla \times\left(\nabla u_{i}, \nabla u_{j}\right)=\mathbf{0} \quad(i, j=1, \cdots, n) \\
& \nabla \cdot\left(\nabla \times\left(\boldsymbol{F}_{1}, \boldsymbol{F}_{2}\right)\right)=0
\end{aligned}
$$




$$
\begin{aligned}
& \nabla \times\left(\left[\boldsymbol{F}_{1} \boldsymbol{F}_{2} \boldsymbol{F}_{3}\right], \boldsymbol{F}_{4}\right)=-\left(\boldsymbol{F}_{3} \cdot \boldsymbol{F}_{4}\right)\left[\boldsymbol{F}_{2}\left(\nabla \cdot \boldsymbol{F}_{1}\right)-\boldsymbol{F}_{1}\left(\nabla \cdot \boldsymbol{F}_{2}\right)\right] \\
& +\left(\boldsymbol{F}_{2} \cdot \boldsymbol{F}_{4}\right)\left[\boldsymbol{F}_{3}\left(\nabla \cdot \boldsymbol{F}_{1}\right)-\boldsymbol{F}_{1}\left(\nabla \cdot \boldsymbol{F}_{3}\right)\right]-\left(\boldsymbol{F}_{1} \cdot \boldsymbol{F}_{4}\right)\left[\boldsymbol{F}_{3}\left(\nabla \cdot \boldsymbol{F}_{2}\right)-\boldsymbol{F}_{2}\left(\nabla \cdot \boldsymbol{F}_{3}\right)\right] \\
& \nabla \times\left(\nabla \times\left(\boldsymbol{F}_{1}, \boldsymbol{F}_{2}\right), \boldsymbol{F}_{3}\right)=-\left(\nabla \cdot \boldsymbol{F}_{3}\right)\left[\boldsymbol{F}_{2}\left(\nabla \cdot \boldsymbol{F}_{1}\right)-\boldsymbol{F}_{1}\left(\nabla \cdot \boldsymbol{F}_{2}\right)\right] \\
& +\left(\boldsymbol{F}_{2} \cdot \boldsymbol{F}_{3}\right)\left[\nabla\left(\nabla \cdot \boldsymbol{F}_{1}\right)-\nabla^{2} \boldsymbol{F}_{1}\right]-\left(\boldsymbol{F}_{1} \cdot \boldsymbol{F}_{3}\right)\left[\nabla\left(\nabla \cdot \boldsymbol{F}_{2}\right)-\nabla^{2} \boldsymbol{F}_{2}\right]
\end{aligned}
$$

All vector functions present in these properties are such as in formulas (5) to (9). It properties as above has a clear parallel with the formulas involving the curl presented in [2]. Demonstrations (P4) to (P8) are elementary, simply by the development of both members of the equations. To check the properties (P9) and (P10), one must show first the following identity relating five vectors in space $I R^{4}$ :

PROPOSITION Given the vectors $\boldsymbol{A}, \boldsymbol{B}, \boldsymbol{C}, \boldsymbol{D}, \boldsymbol{E}$, then:

$$
\begin{aligned}
{[A \boldsymbol{B}[\boldsymbol{C D E}]]=} & \boldsymbol{C}(\boldsymbol{A} \cdot \boldsymbol{E})(\boldsymbol{B} \cdot \boldsymbol{D})+\boldsymbol{D}(\boldsymbol{A} \cdot \boldsymbol{C})(\boldsymbol{B} \cdot \boldsymbol{E})+\boldsymbol{E}(\boldsymbol{A} \cdot \boldsymbol{D})(\boldsymbol{B} \cdot \boldsymbol{C})-\boldsymbol{C}(\boldsymbol{A} \cdot \boldsymbol{D})(\boldsymbol{B} \cdot \boldsymbol{E}) \\
& -\boldsymbol{D}(\boldsymbol{A} \cdot \boldsymbol{E})(\boldsymbol{B} \cdot \boldsymbol{C})-\boldsymbol{E}(\boldsymbol{A} \cdot \boldsymbol{C})(\boldsymbol{B} \cdot \boldsymbol{D})
\end{aligned}
$$

PROOF: If $\boldsymbol{A}=m_{A} \hat{e}_{1}+n_{A} \hat{e}_{2}+p_{A} \hat{e}_{3}+q_{A} \hat{e}_{4}, \quad \boldsymbol{B}=m_{B} \hat{e}_{1}+n_{B} \hat{e}_{2}+p_{B} \hat{e}_{3}+q_{B} \hat{e}_{4}, \quad \boldsymbol{C}=m_{C} \hat{e}_{1}+n_{C} \hat{e}_{2}+p_{C} \hat{e}_{3}+q_{C} \hat{e}_{4}$, $\boldsymbol{D}=m_{D} \hat{e}_{1}+n_{D} \hat{e}_{2}+p_{D} \hat{e}_{3}+q_{D} \hat{e}_{4}, \boldsymbol{E}=m_{E} \hat{e}_{1}+n_{E} \hat{e}_{2}+p_{E} \hat{e}_{3}+q_{E} \hat{e}_{4}$, then:

(a) $[\boldsymbol{C D E}]=\left|\begin{array}{llll}\hat{e}_{1} & \hat{e}_{2} & \hat{e}_{3} & \hat{e}_{4} \\ m_{C} & n_{C} & p_{C} & q_{C} \\ m_{D} & n_{D} & p_{D} & q_{D} \\ m_{E} & n_{E} & p_{E} & q_{E}\end{array}\right|=M \hat{e}_{1}+N \hat{e}_{2}+P \hat{e}_{3}+Q \hat{e}_{4}$,

being $M=\left|\begin{array}{lll}n_{C} & p_{C} & q_{C} \\ n_{D} & p_{D} & q_{D} \\ n_{E} & p_{E} & q_{E}\end{array}\right|, \quad N=-\left|\begin{array}{lll}m_{C} & p_{C} & q_{C} \\ m_{D} & p_{D} & q_{D} \\ m_{E} & p_{E} & q_{E}\end{array}\right|, \quad P=\left|\begin{array}{lll}m_{C} & n_{C} & q_{C} \\ m_{D} & n_{D} & q_{D} \\ m_{E} & n_{E} & q_{E}\end{array}\right|$ and $Q=-\left|\begin{array}{lll}m_{C} & n_{C} & p_{C} \\ m_{D} & n_{D} & p_{D} \\ m_{E} & n_{E} & p_{E}\end{array}\right|$, developed according to the definition (1);

(b) $[\boldsymbol{A} \boldsymbol{B}[\boldsymbol{C D E}]]=\left|\begin{array}{cccc}\hat{e}_{1} & \hat{e}_{2} & \hat{e}_{3} & \hat{e}_{4} \\ m_{A} & n_{A} & p_{A} & q_{A} \\ m_{B} & n_{B} & p_{B} & q_{B} \\ M & N & P & Q\end{array}\right|$;

Substituting (a) in (b), developing and arranging the terms conveniently:

$$
\begin{aligned}
& {[\boldsymbol{A} \boldsymbol{B}[\boldsymbol{C D} \boldsymbol{E}]]=\left|\begin{array}{llll}
\hat{e}_{1} & \hat{e}_{2} & \hat{e}_{3} & \hat{e}_{4} \\
m_{A} & n_{A} & p_{A} & q_{A} \\
m_{B} & n_{B} & p_{B} & q_{B} \\
M & N & P & Q
\end{array}\right|} \\
& =\left(m_{A} m_{E}+n_{A} n_{E}+p_{A} p_{E}+q_{A} q_{E}\right)\left(m_{B} m_{D}+n_{B} n_{D}+p_{B} p_{D}+q_{B} q_{D}\right)\left(m_{C} \hat{e}_{1}+n_{C} \hat{e}_{2}+p_{C} \hat{e}_{3}+q_{C} \hat{e}_{4}\right) \\
& +\left(m_{A} m_{C}+n_{A} n_{C}+p_{A} p_{C}+q_{A} q_{C}\right)\left(m_{B} m_{E}+n_{B} n_{E}+p_{B} p_{E}+q_{B} q_{E}\right)\left(m_{D} \hat{e}_{1}+n_{D} \hat{e}_{2}+p_{D} \hat{e}_{3}+q_{D} \hat{e}_{4}\right) \\
& +\left(m_{A} m_{D}+n_{A} n_{D}+p_{A} p_{D}+q_{A} q_{D}\right)\left(m_{B} m_{C}+n_{B} n_{C}+p_{B} p_{C}+q_{B} q_{C}\right)\left(m_{E} \hat{e}_{1}+n_{E} \hat{e}_{2}+p_{E} \hat{e}_{3}+q_{E} \hat{e}_{4}\right) \\
& -\left(m_{A} m_{D}+n_{A} n_{D}+p_{A} p_{D}+q_{A} q_{D}\right)\left(m_{B} m_{E}+n_{B} n_{E}+p_{B} p_{E}+q_{B} q_{E}\right)\left(m_{C} \hat{e}_{1}+n_{C} \hat{e}_{2}+p_{C} \hat{e}_{3}+q_{C} \hat{e}_{4}\right) \\
& -\left(m_{A} m_{E}+n_{A} n_{E}+p_{A} p_{E}+q_{A} q_{E}\right)\left(m_{B} m_{C}+n_{B} n_{C}+p_{B} p_{C}+q_{B} q_{C}\right)\left(m_{D} \hat{e}_{1}+n_{D} \hat{e}_{2}+p_{D} \hat{e}_{3}+q_{D} \hat{e}_{4}\right) \\
& -\left(m_{A} m_{C}+n_{A} n_{C}+p_{A} p_{C}+q_{A} q_{C}\right)\left(m_{B} m_{D}+n_{B} n_{D}+p_{B} p_{D}+q_{B} q_{D}\right)\left(m_{E} \hat{e}_{1}+n_{E} \hat{e}_{2}+p_{E} \hat{e}_{3}+q_{E} \hat{e}_{4}\right) \\
& =\boldsymbol{C}(\boldsymbol{A} \cdot \boldsymbol{E})(\boldsymbol{B} \cdot \boldsymbol{D})+\boldsymbol{D}(\boldsymbol{A} \cdot \boldsymbol{C})(\boldsymbol{B} \cdot \boldsymbol{E})+\boldsymbol{E}(\boldsymbol{A} \cdot \boldsymbol{D})(\boldsymbol{B} \cdot \boldsymbol{C}) \\
& -\boldsymbol{C}(\boldsymbol{A} \cdot \boldsymbol{D})(\boldsymbol{B} \cdot \boldsymbol{E})-\boldsymbol{D}(\boldsymbol{A} \cdot \boldsymbol{E})(\boldsymbol{B} \cdot \boldsymbol{C})-\boldsymbol{E}(\boldsymbol{A} \cdot \boldsymbol{C})(\boldsymbol{B} \cdot \boldsymbol{D})(\mathrm{QED})
\end{aligned}
$$

From (12), and considering the product of vectors written in the order $[\boldsymbol{A}[\boldsymbol{C D E}] \boldsymbol{B}]=-[\boldsymbol{A B}[\boldsymbol{C D E}]]$ : 
By making the identifications $\left\{\begin{array}{l}\boldsymbol{A} \rightarrow \nabla \\ \boldsymbol{B} \rightarrow \boldsymbol{F}_{4} \\ \boldsymbol{C} \rightarrow \boldsymbol{F}_{1} \\ \boldsymbol{D} \rightarrow \boldsymbol{F}_{2} \\ \boldsymbol{E} \rightarrow \boldsymbol{F}_{3}\end{array}\right.$, one obtains immediately the formula (P9);
By making the identifications $\left\{\begin{array}{l}\boldsymbol{A} \rightarrow \nabla \\ \boldsymbol{B} \rightarrow \boldsymbol{F}_{3} \\ \boldsymbol{C} \rightarrow \nabla \\ \boldsymbol{D} \rightarrow \boldsymbol{F}_{1} \\ \boldsymbol{E} \rightarrow \boldsymbol{F}_{2}\end{array}\right.$, one obtains immediately the formula (P10).

\section{Geometric Interpretations for Vector $\Psi$}

For convenience of notation, we identify the four spatial coordinates in $I R^{4}$ by $x_{1}=x, x_{2}=y, x_{3}=z$ and $x_{4}=h$.

\subsection{The Angular Velocity}

Consider, in $I R^{3}$, the velocity $\boldsymbol{v}=(m, n, p)$ applied to a point $P$ in space, determined by the position vector $\boldsymbol{r}=(x, y, z)$, so that the point $P$ perform a plan rotational motion with constant frequency. The angular velocity of this rotational motion is $\boldsymbol{\omega}=\left(\omega_{1}, \omega_{2}, \omega_{3}\right)$.

Since the three vectors are related in the equation $\boldsymbol{v}=\boldsymbol{\omega} \times \boldsymbol{r}$, is simple to demonstrate the well-known relation $\nabla \times v=2 \omega$, in other words, the curl of velocity $v=(m, n, p)$ is a vector collinear to the vector $\boldsymbol{\omega}=\left(\omega_{1}, \omega_{2}, \omega_{3}\right)$, and twice the norm.

The idea geometric of rotation will be extended to a space of four dimensions. Consider, therefore, the vector $\boldsymbol{v}=(m, n, p, q)$ applied to a point $P$ of the four-dimensional space, determined by the vector $\boldsymbol{r}=(x, y, z, h)$, so that the point $P$ also perform a plan rotational motion with constant frequency. The angular velocity of this rotational motion is $\boldsymbol{\omega}=\left(\omega_{1}, \omega_{2}, \omega_{3}, \omega_{4}\right)$.

The objective is to define a geometrical framework that relates the vectors $v, \omega$ and $r$ for the $I R^{4}$, which is analogous to existing framework in $I R^{3}$. The concept of rotation is presented here in a narrow sense. For a broader approach is recommended to consult $[3,4]$.

According to [1], to obtain the vector $\boldsymbol{v}$ from the vectors $\boldsymbol{\omega}$ and $\boldsymbol{r}$ in $I R^{4}$, it is necessary to have a fourth vector. Given the nature of rotation as circular with constant frequency, the vectors $v, \omega$ and $\boldsymbol{r}$ are two by two perpendicular.

Let us introduce a vector $\hat{\psi}=(a, b, c, d)$ having the following properties:

A) $\hat{\psi}$ is perpendicular simultaneously to the three vectors $\boldsymbol{v}, \boldsymbol{\omega}$ and $\boldsymbol{r}$;

B) $\hat{\psi}$ is a unit vector;

C) $\hat{\psi}$ is a dimensionless vector.

These restrictions are intended:

1) Simplify the proportionality constant that relates the norms of the vectors involved in the product. In this case, by (4), $k=1$.

2) Do not change the relationship $|\boldsymbol{v}|=|\boldsymbol{\omega}||\boldsymbol{r}|$;

3) Make the equation of the previous item remains dimensionally consistent.

The components of the vector $\hat{\psi}$ are obtained from the solution of the system (e1)-(e4), modeled on the arguments presented above:

(e1) $\boldsymbol{v} \cdot \hat{\boldsymbol{\psi}}=0 \Leftrightarrow m \cdot a+n \cdot b+p \cdot c+q \cdot d=0$

(e2) $\boldsymbol{r} \cdot \hat{\boldsymbol{\psi}}=0 \Leftrightarrow x \cdot a+y \cdot b+z \cdot c+h \cdot d=0$

(e3) $\boldsymbol{\omega} \cdot \hat{\boldsymbol{\psi}}=0 \Leftrightarrow \omega_{1} \cdot a+\omega_{2} \cdot b+\omega_{3} \cdot c+\omega_{4} \cdot d=0$

(e4) $|\hat{\psi}|=1 \Leftrightarrow a^{2}+b^{2}+c^{2}+d^{2}=1$

The solution to this system of equations is given by: 


$$
\hat{\psi}=\left(a=-\frac{A_{x}}{\Theta}, b=\frac{A_{y}}{\Theta}, c=-\frac{A_{z}}{\Theta}, d=\frac{A_{h}}{\Theta}\right),
$$

where:

$$
\begin{aligned}
A_{x} & =\left|\begin{array}{ccc}
n & p & q \\
\omega_{2} & \omega_{3} & \omega_{4} \\
y & z & h
\end{array}\right|, \\
A_{y} & =\left|\begin{array}{ccc}
m & p & q \\
\omega_{1} & \omega_{3} & \omega_{4} \\
x & z & h
\end{array}\right|, \\
A_{z} & =\left|\begin{array}{ccc}
m & n & q \\
\omega_{1} & \omega_{2} & \omega_{4} \\
x & y & h
\end{array}\right|, \\
A_{h} & =\left|\begin{array}{ccc}
m & n & p \\
\omega_{1} & \omega_{2} & \omega_{3} \\
x & y & z
\end{array}\right|, \\
\Theta & =\sqrt{A_{x}^{2}+A_{y}^{2}+A_{z}^{2}+A_{h}^{2}} .
\end{aligned}
$$

Thus, the vector $\hat{\psi}=(a, b, c, d)$ can be noted more compactly:

$$
\hat{\psi}=-\frac{1}{\Theta}\left(A_{x},-A_{y}, A_{z},-A_{h}\right),
$$

or

$$
\hat{\boldsymbol{\psi}}=-\frac{1}{\Theta}\left|\begin{array}{cccc}
\hat{e}_{1} & \hat{e}_{2} & \hat{e}_{3} & \hat{e}_{4} \\
m & n & p & q \\
\omega_{1} & \omega_{2} & \omega_{3} & \omega_{4} \\
x & y & z & h
\end{array}\right|,
$$

or more simply:

$$
\hat{\psi}=-\frac{1}{\Theta}[v \omega r]
$$

It is interesting to note that the vector $\Phi=[\boldsymbol{v} \boldsymbol{\omega} \boldsymbol{r}]$ has the dimensions $\mathrm{L}^{2} \cdot \mathrm{T}^{-2}$.

With these definitions and results, the vector $\boldsymbol{v}=(m, n, p, q)$ is obtained from the equation:

$$
\boldsymbol{v}=[\boldsymbol{\omega r} \hat{\boldsymbol{\psi}}]=\left|\begin{array}{cccc}
\hat{e}_{1} & \hat{e}_{2} & \hat{e}_{3} & \hat{e}_{4} \\
\omega_{1} & \omega_{2} & \omega_{3} & \omega_{4} \\
x & y & z & h \\
a & b & c & d
\end{array}\right|,
$$

and the components of the vector $\boldsymbol{v}$ are given by:

$$
\begin{aligned}
& m=\left(\omega_{4} c-\omega_{3} d\right) y+\left(\omega_{2} d-\omega_{4} b\right) z+\left(\omega_{3} b-\omega_{2} c\right) h, \\
& n=\left(\omega_{3} d-\omega_{4} c\right) x+\left(\omega_{4} a-\omega_{1} d\right) z+\left(\omega_{1} c-\omega_{3} a\right) h, \\
& p=\left(\omega_{4} b-\omega_{2} d\right) x+\left(\omega_{1} d-\omega_{4} a\right) y+\left(\omega_{2} a-\omega_{1} b\right) h,
\end{aligned}
$$




$$
q=\left(\omega_{2} c-\omega_{3} b\right) x+\left(\omega_{3} a-\omega_{1} c\right) y+\left(\omega_{1} b-\omega_{2} a\right) z .
$$

The components $(m, n, p, q)$ of $\boldsymbol{v}$ are determined simultaneously with the components $(a, b, c, d)$ of $\hat{\psi}$. Note also that:

$$
\left.\begin{array}{c}
\hat{\psi}=-\frac{1}{\Theta}[\boldsymbol{v} \omega r] \\
|\boldsymbol{v}|=|\boldsymbol{\omega}||\boldsymbol{r}|
\end{array}\right\} \Rightarrow|\hat{\psi}|=\frac{1}{\Theta}|\boldsymbol{v}||\boldsymbol{|}||\boldsymbol{r}|=1 \Rightarrow|\boldsymbol{v}||\boldsymbol{|}||\boldsymbol{r}|=\Theta \Rightarrow|\boldsymbol{v}||\boldsymbol{v}|=\Theta \Rightarrow \Theta=|\boldsymbol{v}|^{2} \Rightarrow \Theta=\boldsymbol{v} \cdot \boldsymbol{v}
$$

that is,

$$
\Theta=v \cdot v .
$$

\subsection{Relationship between the Vectors $\omega$ and $\Psi=\nabla \times(v, \hat{\psi})$}

Consider the analogous to the curl vector, given by

$$
\Psi=\nabla \times(\boldsymbol{v}, \hat{\boldsymbol{\psi}})=\left|\begin{array}{cccc}
\hat{e}_{1} & \hat{e}_{2} & \hat{e}_{3} & \hat{e}_{4} \\
\frac{\partial}{\partial x} & \frac{\partial}{\partial y} & \frac{\partial}{\partial z} & \frac{\partial}{\partial h} \\
m & n & p & q \\
a & b & c & d
\end{array}\right|=\Psi_{1} \hat{e}_{1}+\Psi_{2} \hat{e}_{2}+\Psi_{3} \hat{e}_{3}+\Psi_{4} \hat{e}_{4}
$$

By using relations (23)-(26), shows that:

$\Psi_{1}=2 \omega_{1}, \quad \Psi_{2}=2 \omega_{2}, \quad \Psi_{3}=2 \omega_{3}, \quad \Psi_{4}=2 \omega_{4}$, so that

$$
\nabla \times(v, \hat{\psi})=2 \omega .
$$

This result shows that the relationship between curl and angular velocity remains the same, when using the vector analogous to the curl, in four-dimensional space. The dimensions of the vectors are also consistent, namely, both have dimensions $\mathrm{T}^{-1}$.

It should be noted that there is no intention to give physical meaning to these equations. Even the dimensional analysis is being treated here purely mathematical levels.

The following shows that the vector $\Psi=\nabla \times(v, \hat{\psi})$ in $I R^{4}$ is reduced in the known cases in $I R^{3}$.

\subsection{Particular Cases}

\subsubsection{Projection onto $I R^{3}$}

a) Usual cross product and curl

Being $\boldsymbol{r}=(x, y, z), \boldsymbol{\omega}=\left(\omega_{1}, \omega_{2}, \omega_{3}\right)$ and $\boldsymbol{v}=(m, n, p)$, are well known the results:

$$
\begin{aligned}
\boldsymbol{v}=\boldsymbol{\omega} \times \boldsymbol{r} & =\left|\begin{array}{ccc}
\hat{e}_{1} & \hat{e}_{2} & \hat{e}_{3} \\
\omega_{1} & \omega_{2} & \omega_{3} \\
x & y & z
\end{array}\right|=\left(\omega_{2} z-\omega_{3} y\right) \hat{e}_{1}+\left(\omega_{3} x-\omega_{1} z\right) \hat{e}_{2}+\left(\omega_{1} y-\omega_{2} x\right) \hat{e}_{3} \\
\nabla \times \boldsymbol{v} & =\left|\begin{array}{ccc}
\hat{e}_{1} & \hat{e}_{2} & \hat{e}_{3} \\
\frac{\partial}{\partial x} & \frac{\partial}{\partial y} & \frac{\partial}{\partial z} \\
m & n & p
\end{array}\right|=\left(\frac{\partial p}{\partial y}-\frac{\partial n}{\partial z}\right) \hat{e}_{1}+\left(\frac{\partial m}{\partial z}-\frac{\partial p}{\partial x}\right) \hat{e}_{2}+\left(\frac{\partial n}{\partial x}-\frac{\partial m}{\partial y}\right) \hat{e}_{3} \\
& =\left(\omega_{1}+\omega_{1}\right) \hat{e}_{1}+\left(\omega_{2}+\omega_{2}\right) \hat{e}_{2}+\left(\omega_{3}+\omega_{3}\right) \hat{e}_{3}=2\left(\omega_{1} \hat{e}_{1}+\omega_{2} \hat{e}_{2}+\omega_{3} \hat{e}_{3}\right) \Rightarrow \nabla \times \boldsymbol{v}=2 \boldsymbol{\omega}
\end{aligned}
$$

b) Curl by analogy

If we consider the vectors $\boldsymbol{r}, \boldsymbol{\omega}$ and $\boldsymbol{v}$ like projections from the space $I R^{4}$ onto space $I R^{3}$, concordant results are obtained as follows:

Consider $\boldsymbol{r}=(x, y, z, 0)[h=0], \boldsymbol{\omega}=\left(\omega_{1}, \omega_{2}, \omega_{3}, 0\right) \quad\left[\omega_{4}=0\right]$ and $\boldsymbol{v}=(m, n, p, 0) \quad[q=0]$. 
It is calculated $a=b=c=0, d=1$, and from the relations (23)-(26) we arrive at $m=\omega_{2} z-\omega_{3} y$, $n=\omega_{3} x-\omega_{1} z, \quad p=\omega_{1} y-\omega_{2} x$. Therefore:

$$
\begin{aligned}
\nabla \times(\boldsymbol{v}, \hat{\boldsymbol{\psi}}) & =\left|\begin{array}{cccc}
\hat{e}_{1} & \hat{e}_{2} & \hat{e}_{3} & \hat{e}_{4} \\
\frac{\partial}{\partial x} & \frac{\partial}{\partial y} & \frac{\partial}{\partial z} & \frac{\partial}{\partial h} \\
m & n & p & 0 \\
0 & 0 & 0 & 1
\end{array}\right|=\left(\frac{\partial p}{\partial y}-\frac{\partial n}{\partial z}\right) \hat{e}_{1}+\left(\frac{\partial m}{\partial z}-\frac{\partial p}{\partial x}\right) \hat{e}_{2}+\left(\frac{\partial n}{\partial x}-\frac{\partial m}{\partial y}\right) \hat{e}_{3}+0 \cdot \hat{e}_{4} \\
& =2\left(\omega_{1}, \omega_{2}, \omega_{3}, 0\right) \Rightarrow \nabla \times(v, \hat{\boldsymbol{\psi}})=2 \omega
\end{aligned}
$$

\subsubsection{Projection onto $I R^{2}$}

c) Usual cross product and curl

Being $\boldsymbol{r}=(x, y, 0), \boldsymbol{\omega}=(0,0, \omega)$ and $\boldsymbol{v}=(m, n, 0)$, are well known the results:

$$
\begin{aligned}
& \boldsymbol{v}=\boldsymbol{\omega} \times \boldsymbol{r}=\left|\begin{array}{ccc}
\hat{e}_{1} & \hat{e}_{2} & \hat{e}_{3} \\
0 & 0 & \omega \\
x & y & 0
\end{array}\right|=-\omega y \hat{e}_{1}+\omega x \hat{e}_{2} \\
& \nabla \times \boldsymbol{v}=\left|\begin{array}{ccc}
\hat{e}_{1} & \hat{e}_{2} & \hat{e}_{3} \\
\frac{\partial}{\partial x} & \frac{\partial}{\partial y} & \frac{\partial}{\partial z} \\
-\omega y & \omega x & 0
\end{array}\right|=-\frac{\partial(\omega x)}{\partial z} \hat{e}_{1}+\frac{\partial(-\omega y)}{\partial z} \hat{e}_{2}+\left(\frac{\partial(\omega x)}{\partial x}-\frac{\partial(-\omega y)}{\partial y}\right) \hat{e}_{3} \\
&=(\omega+\omega) \hat{e}_{3} \Rightarrow \nabla \times \boldsymbol{v}=2 \omega
\end{aligned}
$$

d) Curl by analogy

If we consider the vectors $r, \omega$ and $v$ like projections from the space $I R^{4}$ onto space $I R^{2}$, concordant results are obtained as follows:

Consider $\boldsymbol{r}=(x, y, 0,0) \quad[z=h=0], \boldsymbol{\omega}=(0,0, \omega, 0) \quad\left[\omega_{1}=\omega_{2}=\omega_{4}=0\right]$ and $\boldsymbol{v}=(m, n, 0,0) \quad[p=q=0]$. It is calculated $a=b=c=0, d=1$, and from the relations (23)-(26) we arrive at $m=-\omega y, n=\omega x$. Therefore:

$$
\begin{aligned}
\nabla \times(\boldsymbol{v}, \hat{\boldsymbol{\psi}}) & =\left|\begin{array}{cccc}
\hat{e}_{1} & \hat{e}_{2} & \hat{e}_{3} & \hat{e}_{4} \\
\frac{\partial}{\partial x} & \frac{\partial}{\partial y} & \frac{\partial}{\partial z} & \frac{\partial}{\partial h} \\
m & n & 0 & 0 \\
0 & 0 & 0 & 1
\end{array}\right|=-\frac{\partial n}{\partial z} \hat{e}_{1}+\frac{\partial m}{\partial z} \hat{e}_{2}+\left(\frac{\partial n}{\partial x}-\frac{\partial m}{\partial y}\right) \hat{e}_{3}+0 \cdot \hat{e}_{4} \\
& =2(0,0, \omega, 0) \Rightarrow \nabla \times(\boldsymbol{v}, \hat{\boldsymbol{\psi}})=2 \omega
\end{aligned}
$$

\section{Conclusions}

Through dimensional analogies and structures based on determinants, we could get a vector field in a space of four dimensions, with algebraic and geometric properties, equivalent to the properties of vector curl commonly defined in $I R^{3}$.

Subsequent developments show that the "curl by analogy" thus obtained reduces to the three-dimensional case when the fourth coordinate is null in their constitutive equations.

The geometric frameworks that relate to the vectors $\boldsymbol{r}, \boldsymbol{\omega}$ and $\boldsymbol{v}$ in a circular rotational motion with constant frequency are equivalent in three and four dimensions, and the appendix of this work also suggests that they are equivalent in any dimension. Discussing the behavior of the vector $\hat{\psi}$ during such rotations will be the subject of future analysis.

This paper is not intended, of course, to give any physical interpretation of the quantities involved, but only to demonstrate the algebraic and geometric analogies related to the rotation in three and four dimensions, including 
the demonstration of consistency with regard to dimensional analysis.

In future work it is intended to apply the results obtained in triads of vectors with similar relationships to those in between $\boldsymbol{r}, \boldsymbol{\omega}$ and $\boldsymbol{v}$, such as, for example, in relation $\boldsymbol{B}=\frac{1}{c} \hat{\boldsymbol{u}} \times \boldsymbol{E}$ between the magnetic induction $\boldsymbol{B}$, electric field $\boldsymbol{E}$, and vector directional of propagation of electromagnetic wave, represented by $\hat{\boldsymbol{u}}$.

\section{REFERENCES}

[1] L. Simal Moreira, "Geometric Analogy and Products of Vectors in $n$ Dimensions," Advances in Linear Algebra \& Matrix Theory, Vol. 3, No. 1, 2013, pp. 1-6. http://dx.doi.org/10.4236/alamt.2013.31001

[2] M. R. Spiegel, S. Lipschutz and J. Liu, “Mathematical Handbook of Formulas and Tables,” 3rd Edition, Schaum's Outline Series, McGraw-Hill, New York, 2009.

[3] F. N. Cole, “On Rotations in Space of Four Dimensions,” American Journal of Mathematics, Vol. 12, No. 2, 1890 , pp. $191-210$. http://www.jstor.org/stable2369715

[4] H. P. Manning, “Geometry of Four Dimensions,” Dover Publications, Mineola, 1956.

\section{Appendix}

There is no difficulty in extending the ideas about rotations presented in this work to higher dimensions, simply just utilize the aforementioned systematic increase of coordinates for points and vectors, as well as rows and columns to the determinants that make up the structures generating products of vectors [1]. Indeed, for any dimension $n$ would:

$$
\boldsymbol{v}_{(n)}=\left[\omega \boldsymbol{r} \hat{\boldsymbol{\psi}}_{1} \hat{\boldsymbol{\psi}}_{2} \cdots \hat{\boldsymbol{\psi}}_{n-3}\right],
$$

or

And also:

$$
\boldsymbol{v}_{(n)}=\left|\begin{array}{cccccc}
\omega_{1} & \omega_{2} & \omega_{3} & \omega_{4} & \cdots & \omega_{n} \\
x_{1} & x_{2} & x_{3} & x_{4} & \cdots & x_{n} \\
a_{11} & a_{12} & a_{13} & a_{14} & \cdots & a_{1 n} \\
a_{21} & a_{22} & a_{23} & a_{24} & \cdots & a_{2 n} \\
\vdots & \vdots & \vdots & \vdots & \ddots & \vdots \\
a_{n-3,1} & a_{n-3,2} & a_{n-3,3} & a_{n-3,4} & \cdots & a_{n-3, n}
\end{array}\right| .
$$

or

$$
\Psi_{(n)}=\nabla \times\left(\boldsymbol{v}, \hat{\boldsymbol{\psi}}_{1}, \hat{\boldsymbol{\psi}}_{2}, \cdots, \hat{\boldsymbol{\psi}}_{n-3}\right),
$$

$$
\Psi_{(n)}=\left|\begin{array}{cccccc}
\hat{e}_{1} & \hat{e}_{2} & \hat{e}_{3} & \hat{e}_{4} & \cdots & \hat{e}_{n} \\
\frac{\partial}{\partial x_{1}} & \frac{\partial}{\partial x_{2}} & \frac{\partial}{\partial x_{3}} & \frac{\partial}{\partial x_{4}} & \cdots & \frac{\partial}{\partial x_{n}} \\
v_{1} & v_{2} & v_{3} & v_{4} & \cdots & v_{n} \\
a_{11} & a_{12} & a_{13} & a_{14} & \cdots & a_{1 n} \\
\vdots & \vdots & \vdots & \vdots & \ddots & \vdots \\
a_{n-3,1} & a_{n-3,2} & a_{n-3,3} & a_{n-3,4} & \cdots & a_{n-3, n}
\end{array}\right| .
$$

